\title{
Ring Pessary Sizer: a pilot study to objectively measure size of a ring pessary required by a patient
}

\author{
N. S. Qureshi • F. Appleton • A. B. Jones
}

Received: 24 October 2007 / Accepted: 30 November 2007 / Published online: 4 January 2008

(C) Springer-Verlag 2007

\begin{abstract}
Ring pessaries are frequently used to relieve the symptoms of utero-vaginal prolapse. So far, the assessment of the size of a ring pessary required by a patient has been subjective. We have developed an instrument, called a 'Ring Pessary Sizer', which could objectively estimate the size of ring required by a patient. This article details a prospective study of 37 patients who were using ring pessary without any problems for whom the Ring Pessary Sizer was able to correctly estimate within one size of the ring in 31 (84\%) cases. A Ring Pessary Sizer would make the choice of the size of a ring pessary more objective for a clinician and less inconvenient for a patient. This will also have numerous cost saving benefits.
\end{abstract}

Keywords Ring pessary · Utero-vaginal prolapse

\section{Introduction}

Pelvic organ prolapse has been described as far back as $1800 \mathrm{BC}$ [1]. Throughout history there have been a number of references to the use of pessaries to relieve the symptoms of utero-vaginal prolapse. It has been estimated that up to $20 \%$ of women by the age of 60 would be affected by the condition [2]. Based on projection, the number of women over the age of 65 will double in the next 25 years [3], and

\footnotetext{
N. S. Qureshi $(\square)$

Department of Obstetrics and Gynaecology,

Royal Surrey County Hospital,

Guildford GU2 7XX, UK

e-mail: nsq@doctors.net.uk

F. Appleton · A. B. Jones

Gynaecology Department, Wrexham Maelor Hospital,

Wrexham LL13 7TD, UK
}

hence more cases of utero-vaginal prolapse will be seen. Despite the improvement in medical technology and surgical techniques, which have been used as the mainstay treatment for utero-vaginal prolapse, on average 132,000 ring pessaries are sold by one manufacturer in the UK every year (Smith Medical, Kent, United Kingdom, 2005, personnel communication). To determine the size of ring pessary required by the patient, a digital assessment of distance between symphysis pubis (SP) and posterior fornix (PF) of the vagina is made. Trial and error is regarded as the only method to determine the proper size of the ring pessary [4]. We have developed an instrument, called the Ring Pessary Sizer (Fig. 1), which would correctly determine the SP to PF distance and vaginal width in a single reading.

The aim of this pilot study was to explore the potential of the Ring Pessary Sizer (RPS) in measurement of a ring required by the patient.

\section{Methods}

This prospective trial studied gynaecology outpatients of Wrexham Maelor Hospital, and had the North East Wales research ethics committee approval. The study was performed on patients who had already been using ring pessaries for more than a year without any problems to relieve the symptoms of utero-vaginal prolapse. Patients with language problems and with special needs were excluded from the study. The patients using ring pessaries were identified from the database of the outpatients and were sent an appointment to specially set up 'ring pessary clinics' for the purpose of the study along with information leaflets about the trial. Following counselling and informed consent, the patient was placed in a dorsal position for a 
Fig. 1 Ring Pessary Sizer

gynaecological examination. The investigators (NSQ or FA) were not told the size of ring a patient was using. The old ring was removed by one of the authors (NSQ or FA), and the RPS was used to determine the size of ring pessary. The instrument is graduated with the sizes of the ring pessary. These are $3 \mathrm{~mm}$ intervals from 53 to $80 \mathrm{~mm}$, and $5 \mathrm{~mm}$ intervals from 80 to $110 \mathrm{~mm}$. The RPS was gently introduced into the vagina (no lubricant required) and was directed towards the PF of the vagina. A vaginal examination may be performed at the time of introduction of RPS into the vagina to ensure that PF is reached. Once the distal end of the Ring Pessary Sizer had reached the PF, the sliding lock over the instrument slid gently with the index finger of the examining hand to the SP. This showed the size of ring pessary required by the patient (Fig. 2). The same size ring pessary was replaced which the patient had been wearing before.

The main objective of this study was to assess the extent of agreement between the size currently used and that suggested by RPS.

Bland-Altman analyses of the findings were performed using StatsDirect statistical software (http:/www.statsdirect. $\mathrm{com} /$ ).

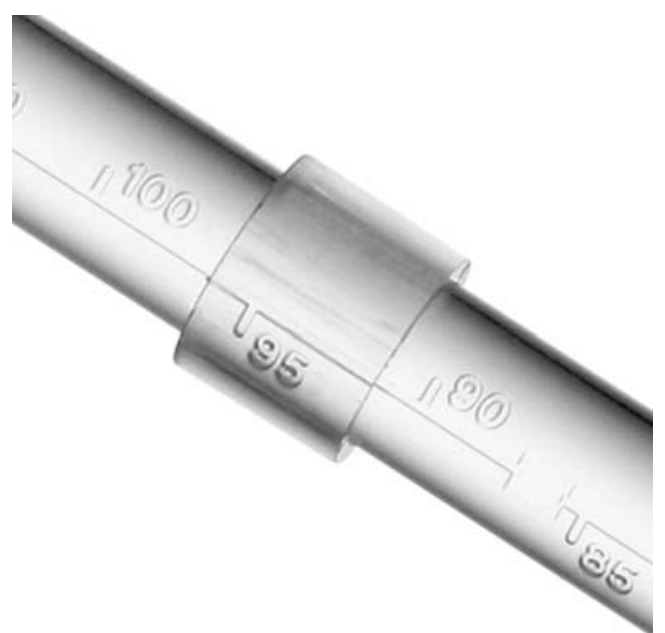

Fig. 2 Ring Pessary Sizer showing size of ring pessary required by a patient

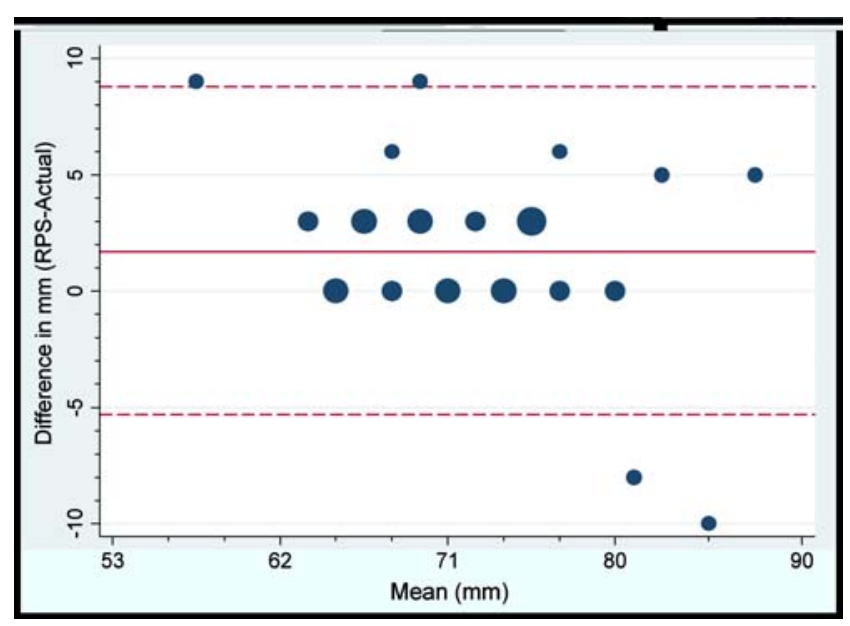

Fig. 3 Bland-Altman analysis of difference between actual and estimated ring size by the Ring Pessary Sizer. RPS Ring Pessary Sizer

\section{Results}

A total of 37 patients were recruited to the study. The age range of the patients was 59-87 years and parity was between 1 and 6 . Twenty-nine (78\%) patients were assessed by NSQ and eight (22\%) by FA. Of the 37 patients, the RPS exactly estimated the ring size in $14(38 \%)$ cases and within one ring size in $31(84 \%)$ cases. Of the patients using ring sizes between 59 and $80 \mathrm{~mm}$, the RPS correctly estimated within one size in $34(92 \%)$ cases. The results are shown in Fig. 3.

\section{Discussion}

The results of this study demonstrate the usefulness of RPS in determining the size of a ring pessary required by a patient. The RPS had been most accurate in determining ring sizes between 59 and $80 \mathrm{~mm}(<59 \mathrm{~mm}$ ring size was overestimated three sizes and $>80 \mathrm{~mm}$ size it was underestimated two sizes). A possible explanation for this could be the changes in pelvic floor muscles in patients requiring smaller $(<59 \mathrm{~mm})$ or lager size $(>80 \mathrm{~mm})$ rings. Therefore, while making an assessment for a pessary size for a patient, the softness of the connective tissue of the paravaginal area and the laxity of the pelvic floor muscles should also be considered before determining a ring size.

Neither assessor (NSQ, FA) experienced difficulty in the use of RPS and obtaining readings for a ring pessary size. The possibility of personal bias in the study to determine the ring pessary size by the RPS is extremely low as it is not easy to correctly determine the ring pessary size by just looking at it after it was removed from the patient.

Ring pessaries are commonly used in gynaecology outpatient settings. A study has showed that $86 \%$ of gynaecologists and $98 \%$ of urogynaecologists use them 
[5]. The main indications for the use of ring pessary are the symptoms of utero-vaginal prolapse and urinary symptoms. Vaginal pessaries are the only currently available nonsurgical intervention for managing women with a prolapse. Conservative management is advised for patients who are not fit for surgery or do not want surgery. In a prospective study of 100 consecutive women with symptomatic pelvic organ prolapse fitted with a pessary, 73 women retained the pessary 2 weeks later. After 2 months, $92 \%$ of these women were satisfied with the pessary; virtually all symptoms of prolapse and $50 \%$ of urinary symptoms had resolved, although occult stress incontinence was unmasked in $21 \%$ of the women [6].

Ring pessaries are relatively inexpensive items, the average cost of a ring pessary is $£ 1.20$. However, the RPS is cheaper than the cost of a single ring pessary. The subjective method of finding an appropriate size of ring pessary may be uncomfortable for patient and causes wastage of unused opened rings. Although, 'falling out' of ring pessaries is not only due to incorrect measurement of ring size but may also be due to laxity of pelvic floor muscle [7]. However, a RPS adds a degree of objectivity in determining a ring size for a patient. Ill-fitted rings often fall out and patients have to be seen as outpatients again, causing inconvenience to patients and putting an unnecessary burden on the running of clinics. The cost of trying various sizes of ring pessaries on the emotional well-being of patients is immeasurable.

The use of the RPS may not be necessary for a trained gynaecologist to determine the size of ring required by the patient. However, in larger hospitals where there is a trend to refer this procedure to 'ring clinics', availability of the RPS would be extremely useful. The 'ring clinics' are usually run by nurse practitioners who may be relatively inexperienced in digitally determining the size of a ring accurately. Although, a gynaecologist specifying the size of a ring pessary may initially make referral to 'ring clinics'. It is a well-known phenomenon that the size of a ring pessary may change with age [8].

The use of a RPS to determine the size of ring pessary required by a patient is a straightforward procedure. We feel that the learning curve for nurses/nurse practitioners who have experience in performing Cusco's speculum examination to use the Ring Pessary Sizer would be steep and that supervision by a gynaecologist in the first five cases may be required.

We intend to perform a larger, randomised controlled study in the future to either have the ring pessaries fitted using digital assessment or by the Ring Pessary Sizer with follow-up which would include time to fit, number of wasted ring pessaries, and requirement for subsequent refittings.

We feel that introduction of the RPS to gynaecology outpatients will assist the clinician and improve the outcome of this procedure for a patient. This in itself will prove beneficial and cost effective to Health Trusts throughout the United Kingdom.

Acknowledgement The study had North East Wales Local Research Ethics Committee approval (06/Wno03/41). The authors are very grateful to the nursing staff of the gynaecology outpatients. We are also thankful to all the consultants in the Department of Obstetrics and Gynaecology at the hospital for giving permission to recruit their patients into the trial and to Andy Vail for his help in the statistical analysis.

Disclosure of interests NSQ is the inventor and holds the UK patent for the Ring Pessary Sizer.

\section{References}

1. Rock JA, Thompson JD (1997) Telinde's operative gynaecology. Lippincott-Raven, Philadelphia, PA, pp 1077-1085

2. Oslen AL, Smith VJ, Bergstrom JO, Colling JC, Clark AL (1997) Epidemiology of surgically managed pelvic organ prolapse and urinary incontinence. Obstet Gynecol 89:501-506

3. Weber AM, Richter HE (2005) Pelvic organ prolapse. Obstet Gynecol 106:615-634

4. Bash KL (2000) Review of vaginal pessaries. Obstet Gynecol Surv $7: 455-460$

5. Cundiff GW, Weidner AC, Visco AG, Bump RC, Addison WA (2000) A survey of pessary use by members of American Urogynecologic Society. Obstet Gynecol 95:931-935

6. Clemon JL, Aguilar VC, Tillingghast TA, Jackson ND, Myers DL (2004) Patient satisfaction and changes in prolapse and urinary symptoms in women who were fitted successfully with a pessary for pelvic organ prolapse. Am J Obstet Gynecol 190:1025-1029

7. Doshani A, Teo REC, Mayne CJ, Tincello DG (2007) Uterine prolapse. Br Med J 335:819-823

8. Bump RC, Mattiasson A, Bø K, Brubaker LP, DeLancy JOL, Klarskov P, Shull BL, Smith ARB (1996) The standardization of terminology of female pelvic organ prolapse and pelvic floor dysfunction. Am J Obstet Gynecol 175:10-17 